\title{
Photoluminescence and electron paramagnetic resonance studies of springlike carbon nanofibers
}

\author{
Bipin Kumar Gupta, ${ }^{\text {a) }}$ V. Shanker, Manju Arora, and D. Haranath \\ National Physical Laboratory (CSIR), Dr. K. S. Krishnan Road, New Delhi 110 012, India
}

(Received 13 June 2009; accepted 25 July 2009; published online 21 August 2009)

\begin{abstract}
Carbon nanofiber (CNF) with springlike and double-helix structures has been synthesized by catalytic thermal pyrolysis of an acetylene precursor at $850-950{ }^{\circ} \mathrm{C}$ using iron nanopowder and thiophene as catalyst and promoter, respectively. High resolution electron microscopy revealed a higher $d$-spacing $(\sim 3.46 \AA)$ of $(002)$ crystal plane than customary multiwalled carbon nanotube (MWCNT) (3.37 ̊̊) that helps in sustaining mechanical shocks better than MWCNTs. The large surface to volume ratio of springlike CNF does provide many delocalized free electrons to enhance the photoluminescence activity. Electron paramagnetic resonance signal showed a single narrow line having $g$-value $2.0024 \pm 0.0002$ and spin contribution $3.4956 \times 10^{-16} \mathrm{spins} / \mathrm{g}$. (C) 2009 American Institute of Physics. [DOI: 10.1063/1.3206663]
\end{abstract}

During past decade, research and development on carbon nanostructures has been focused primarily on the growth control of desired morphologies, their dimensions, and properties for the design of advanced functional materials. ${ }^{1}$ Among them, springlike carbon nanofiber (CNF) is unique due to its shape, functionality, and diversified application potential. One could design many functional materials for the applications involving electromagnetic wave absorber ${ }^{3}$ (frequency range: $8.2-12.4 \mathrm{GHz}$ ) and/or filters, smart tunable microdevices, ${ }^{4}$ microsensors, ${ }^{4}$ three-dimensional composites, ${ }^{5}$ superelastic coils, ${ }^{5}$ biomarkers, ${ }^{5}$ chiral catalyst, ${ }^{5}$ field electron emitters, ${ }^{5}$ hydrogen storage materials, ${ }^{6}$ super capacitor, ${ }^{7}$ and so on.

Springlike CNFs with double-helix structure ${ }^{8}$ have become the object of much interest in biological relevance because similar morphologies do exist between deoxyribonucleic acid and CNFs. Other strategic practices of these materials could be conceived but there are only few research groups $^{9-13}$ that have been working on the growth of coiled carbon filaments for explicit applications. As a general practice, researchers all over the world have been synthesizing microcoiled carbon fibers using thin metal film, metal supported on fine ceramic powder, iron coated indium tin oxide (ITO), thin films of $\mathrm{Fe}, \mathrm{Ni}, \mathrm{Cr}, \mathrm{Ti}, \mathrm{Zn}$, and their oxides coated on silicon or copper substrates; and studied the morphology, growth mode, and elongation lengths of the coiled nanostructures. ${ }^{14-20}$ However, the synthesis of springlike CNFs employing ultrafine metal nanoparticles instead of usual micron-sized particles and observation of surface states by photoluminescence (PL) technique has not been examined so far. It is known that the morphology and size of catalyst are highly responsible for the growth of coiled CNFs. Hence, in the current experiment, we have used polyhedron-shaped iron nanoparticles of size $<100 \mathrm{~nm}$ as a catalyst (as evidenced by scanning electron microscopy observations) and were coated inside the quartz firing tube using acetone as solvent. A cost-effective precursor that produces acetylene gas in the chemical reaction was made to decompose in a preheated $\left(850-950{ }^{\circ} \mathrm{C}\right)$ quartz tube using a spray atomiza-

\footnotetext{
${ }^{\text {a) Electronic addresses: }}$
}

bipinbhu@yahoo.com

and tion setup with a fine nozzle $(\Phi \sim 0.5 \mathrm{~mm})$ attached at one end of the quartz tube. The carrier gas of $90 \% \mathrm{Ar}$ and $10 \%$ $\mathrm{H}_{2}$ (each $99.99 \%$ pure, IOLAR grade) was passed through the spray atomizer containing the acetylene precursor solution and thiophene as a promoter. In our experiment, Ar gas has been used to atomize the solution and $\mathrm{H}_{2}$ gas for maintaining graphitization of the materials throughout the firing tube. The most productive reaction conditions are as follows: temperature $850-950{ }^{\circ} \mathrm{C}$ for $2 \mathrm{~h}$, mixture of $\mathrm{Ar}$ and $\mathrm{H}_{2}$ gas flow rate $\sim 200$ SCCM (SCCM denotes standard cubic centimeter per minute), and the precursor spray rate $\sim 2.5 \mathrm{cc} / \mathrm{min}$. After the reaction, a carbonious material was found to be deposited inside the walls of the quartz tube in the form of ultrafine nanopowder consisting of springlike CNFs with a high degree of homogeneity and reproducibility $>80 \%$.

Figures 1(a) and 1(b) show the representative scanning electron micrographs of springlike and double-helix structures of CNFs. It is observed that the CNFs synthesized at $\sim 850{ }^{\circ} \mathrm{C}$ have a customary springlike structure having an average diameter of the fiber $\sim 90 \mathrm{~nm}$ with the lengths extending in tens of microns. The inset of Fig. 1(a) shows the magnified image of a single springlike CNF. Furthermore, it is very interesting to find out that a petite increase in temperature up to $\sim 900{ }^{\circ} \mathrm{C}$ resulted in double-helix structure as shown in Fig. 1(b). It is inferred that the crossing of pair of CNFs can form a double-helix that grow simultaneously in the same direction from the Fe nanoparticle seed (catalyst). The inset of Fig. 1(b) shows a representative transmission
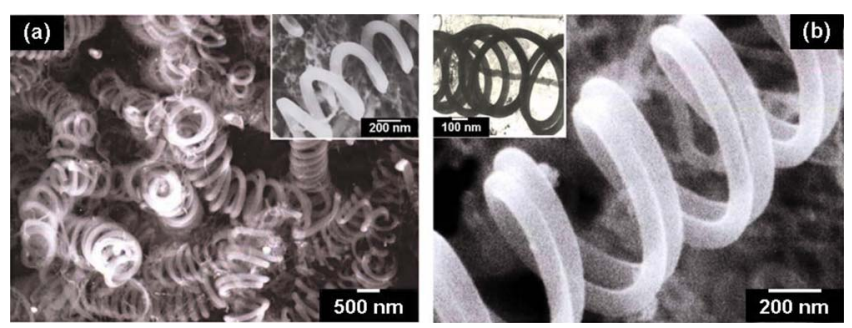

FIG. 1. (Color online) (a) SEM micrograph of as-synthesized springlike CNF structures. The inset shows the magnified image of a single springlike CNF. (b) SEM micrograph of double-helix shaped CNF. The inset shows the TEM image of a double-helix CNF taken at a magnification of $200 \mathrm{kX}$. 


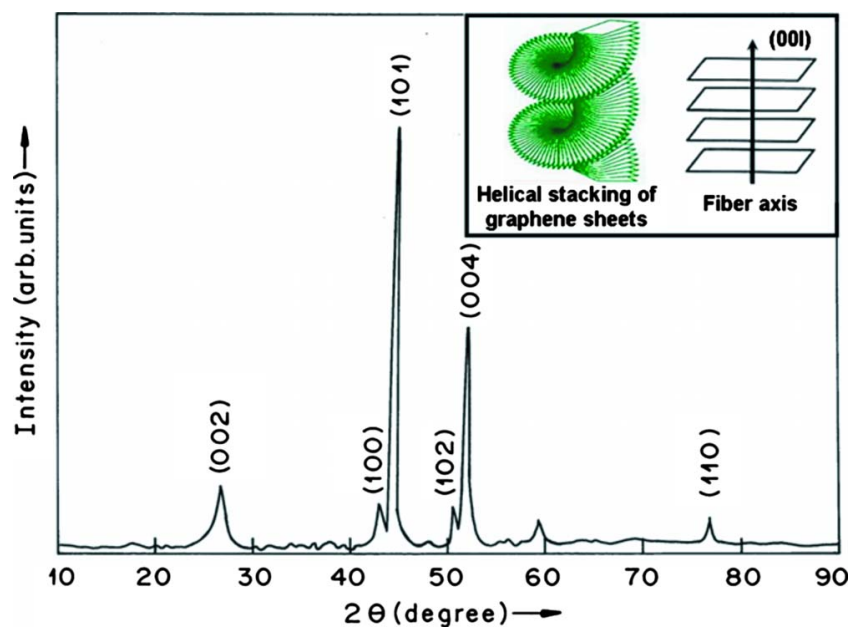

FIG. 2. (Color online) XRD pattern for as-synthesized springlike CNF. The inset shows a preferred orientation of the springlike CNF is along (101) plane.

electron micrograph (TEM) exhibiting a double-helix structure.

In 2002, Motojima and co-workers ${ }^{4,21}$ proposed an acceptable growth mechanism for the carbon microcoils based on the crystal faces of the catalyst grain. Similar analogy could be extended to the present case of springlike CNFs. According to this model, dissolution, diffusion, and precipitation are the three important stages for the formation of CNFs. Initially, the acetylene gas is adsorbed on the surfaces of the polyhedron shaped iron (catalyst) nanoparticles and decomposes to carbon. The carbon atoms then diffuse through the catalyst and precipitates from the lower surfaces of the particle in the form of CNFs. The carbon fibers grow from the faces of the polyhedron shaped iron nanoparticle in the opposite directions and curl to form helical structures caused by the anisotropic deposition of carbon. In other words, one can say that varied diffusion path lengths of carbon atoms in different crystal planes of faceted polyhedron catalyst particles, nanofibers with helical shape are formed. The carbon fibers tend to curl in such a way that the greater anisotropy of the carbon deposition increases the overall spring diameter. It is also considered that the reaction temperature, flow rate of $\mathrm{Ar}$ and $\mathrm{H}_{2}$ gas mixture, and the spray rate of the precursor solution strongly affect the composition of $\mathrm{Fe}-\mathrm{C}-\mathrm{S}-\mathrm{O}$ and thus influence the spring diameter and spring pitch. In other words, higher or lower reaction temperature than the required for reaction kinetics may result in the decrease of anisotropic composition on the respective polyhedron crystal faces. The same effect is observed in case of slow spray rate of acetylene precursor and/or that of flow rate of carrier gases. Finally, it could be documented that if there is no anisotropy between the respective crystal faces, then straight carbon fibers will be formed. All these CNF based nanostructures either springlike, double-helix, or straight carbon fiber find innumerable applications in many technologically advancing areas of science. ${ }^{22-24}$

Figure 2 shows the $\mathrm{X}$-ray diffraction (XRD) pattern for as-synthesized spring-like CNFs. The XRD data shows the peaks corresponding to hexagonal structure ${ }^{25}$ of graphite. No diffraction peaks from the catalyst (iron nanoparticle) or any other impurity were detected because of extremely small quantity of catalyst employed and the purity of our process of preparation. The lattice parameters $a$ and $c$ were estimated

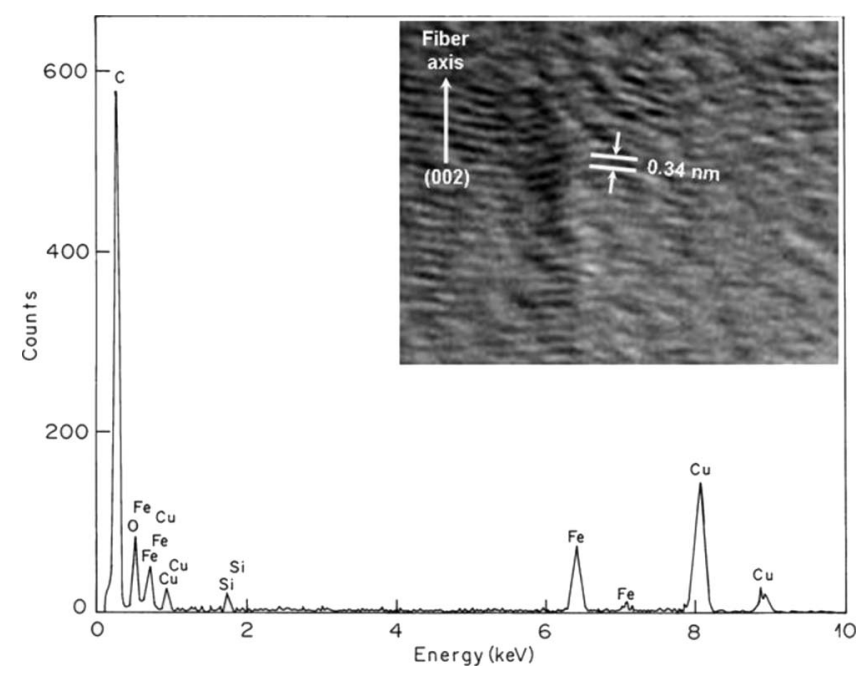

FIG. 3. Energy dispersive $\mathrm{x}$-ray analysis depicting the elemental composition of a springlike CNF. The inset shows the HRTEM of a cross section of a springlike $\mathrm{CNF}$.

to be 2.4613 and $6.4631 \AA$, respectively, using a nonlinear least square fitting method. The XRD peak analysis revealed a strong tendency for the preferred orientation in the assynthesized springlike CNFs with (101) peak of the maximum intensity as illustrated in the inset of Fig. 2. The springlike CNF, (001) planes are of the finite extent $(\sim 100 \mathrm{~nm})$ arranged nearly perpendicular to fiber axis. Thus, the XRD peak profile for (001) plane including (002) plane will show a broadening effect. The other planes such as (101) and (110) do not have finite extension as that of (001) planes and hence, the peaks corresponding to these will not be broaden but exist relatively sharp in nature. ${ }^{26}$

The spot elemental analysis of springlike CNF was done using energy dispersive x-ray analysis, which is shown in Fig. 3. The study revealed the presence of carbon and iron, from the CNF, oxygen from the environment, and copper and silica from the formvar-coated grid. The inset shows high-resolution transmission electron micrograph of a springlike CNF with a higher $d$-spacing $(\sim 3.46 \AA)$ of $(002)$ crystal plane than customary multiwalled carbon nanotube (MWCNT) (3.37 $\AA$ ) that could be useful in sustaining mechanical shocks better than CNTs.

Figure 4 shows the steady-state PL spectra and the inset shows the excitation spectra of springlike CNFs and customary MWCNTs for comparison. The major peak for both CNF and MWCNT are observed to be located at $\sim 394 \mathrm{~nm}$ for an excitation at $246 \mathrm{~nm}$. But it is interesting to note that the PL intensity of $\mathrm{CNF}$ is almost two times stronger than the MWCNT due to higher surface to volume ratio of the former. Since the active surface area is more, the springlike CNF contains more delocalized $\pi$ electrons available at the surface. Upon UV (246 nm) excitation, not only the delocalized $\pi$ electrons from the walls of the spring will be contributing for the electron-hole recombination, but also the electrons from the top and bottom surfaces will be taking part effectively. A large Coulombic coupling between the photoexcited electrons and holes will be created to strongly bound excitons. The probability of a material to exhibit enhanced luminescence obviously depends on its intrinsic band structure and also other internal and external factors. The internal factors are the surface reconstructions, dislocations, dopants, surface and/or bulk defects, and the external factors that in- 


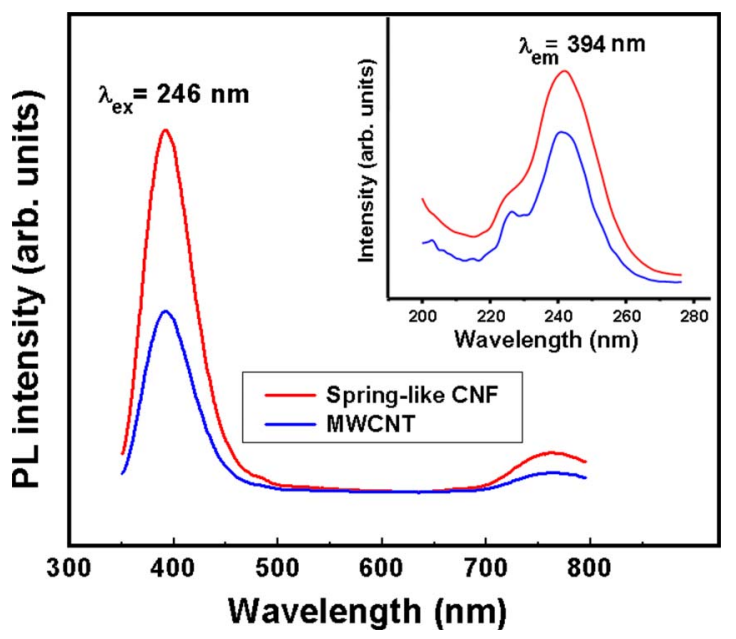

FIG. 4. (Color online) Steady-state PL emission spectra of springlike CNF and customary MWCNT powder recorded at $246 \mathrm{~nm}$ excitation. The inset shows the corresponding PLE spectra of springlike CNF and MWCNT registered at $394 \mathrm{~nm}$ emission.

clude dielectric environment, electromagnetic fields, hydrostatic pressures, etc. These factors may deplete or fill existing defects or change the band structure entirely. As a result, PL emission energies may be altered even to the point of quenching the luminescence altogether depending upon the extent of saturation of the defect states. In the present case of springlike CNFs, the PL emission solely depends on the presence of extensive delocalized $\pi$ electrons in the excited states relaxing to the ground state and the excitation energy trapping associated with the defects. ${ }^{27}$ This signifies the overall enhancement of the PL emission compared to that of MWCNTs.

In order to evaluate the presence of high concentration of delocalized $\pi$ electrons on the surface of springlike CNFs, we carried out the electron paramagnetic resonance (EPR) studies as shown in Fig. 5. EPR involves the resonant absorption of microwaves in the range of $8.8-9.9 \mathrm{GHz}$ by the unpaired electrons of the sample in the presence of magnetic field (range of 100-15000 G) and gives a derivative spectrum. Magnetic field was modulated at $100 \mathrm{kHz}$ and $10 \mathrm{~mW}$ microwave power was used to restrict saturation effects. 1,1 diphenyl 2-picryl hydrazyl was used as a standard field marker for $g$-value and spin density determination. The $g$-value is a dimensionless constant having value 2.002319 and gives information about the electronic structure. ${ }^{28}$ EPR spectrum of springlike CNF showed a strong and narrow Lorentzian line-shaped signal having $g$-value $2.0024 \pm 0.0023$, which is very close to that of a free elec-

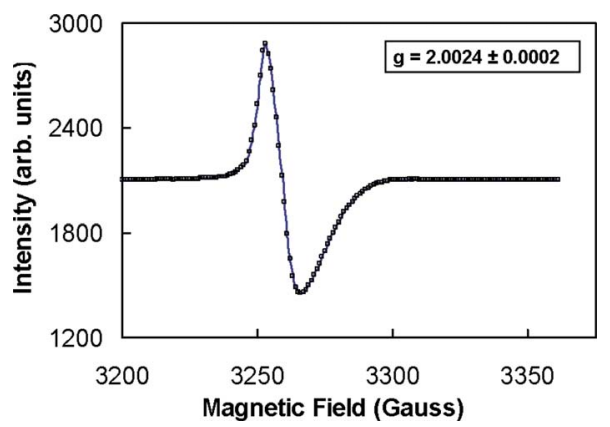

FIG. 5. (Color online) Electron paramagnetic resonance (EPR) spectrum of springlike CNF sample. tron. The appearance of this resonance signal is attributed to the presence of dangling bonds of tetrahedral carbon atoms. ${ }^{28}$ This confirms the presence of delocalized free electrons over the surface of springlike CNF structure. These bonds are formed to relieve the strain energy of springlike CNF structure to gain the stability of the system. The high intensity of the EPR peak is attributed to the large surface area as the majority of the dangling bonds is pointing outside the surface and parallel to each other. The concentration of the dangling bonds has been estimated by a comparison method using area under the curve of the standard and the CNF sample under the same experimental conditions. The concentration of spins has been estimated to be 3.459 $\times 10^{16}$ spins $/ g$, which justifies the argument that the springlike CNF has sufficient delocalized $\pi$ electrons.

In summary, various springlike CNFs have been synthesized using a simple, cost-effective catalytic thermal pyrolysis method involving an acetylene precursor. The luminescence enhancement in spring-like CNFs as compared to MWCNTs is attributed to large surface to volume ratio of the former and many delocalized free electrons available on their surfaces. The EPR signal showed a single narrow line having $g$-value $2.0024 \pm 0.0002$ and spin contribution 3.4956 $\times 10^{-16} \mathrm{spins} / \mathrm{g}$. Springlike CNF structures are highly desirable in designing multifunctional materials for numerous specialized futuristic applications.

${ }^{1}$ L. Meng, C. Fu, and Q. Lu, Prog. Nat. Sci. 19, 801 (2009).

${ }^{2}$ J. Vera-Agullo A. Glória-Pereira, H. Verela-Rizo, J. L. Gonzalez, and I. Martin-Gullon, Compos. Sci. Technol. 69, 1521 (2009).

${ }^{3}$ H. Bi, K.-C. Kou, K. Ostrikov, L.-K. Yan, and Z.-C. Wang, J. Alloys Compd. 478, 796 (2009).

${ }^{4}$ X. Chen, S. Motojima, and H. Iwanaga, J. Cryst. Growth 237-239, 1931 (2002).

${ }^{5}$ J.-H. Kim, A. K. Sharma, and Y.-S. Lee, Mater. Lett. 60, 1697 (2006).

${ }^{6}$ S. K. Beyaz, F. D. Lamari, B. P. Weinverger, P. Gadelle, L. Firlej, and P. Bernier, Int. J. Hydrogen Energy 34, 1965 (2009).

${ }^{7}$ A. G. Pandolfo and A. F. Hollenkamp, J. Power Sources 157, 11 (2006).

${ }^{8}$ S. Motojima, S. Hoshiya, and Y. Hishikawa, Carbon 41, 2658 (2003).

${ }^{9}$ R. T. K. Baker, P. S. Harris, and S. Terry, Nature (London) 253, 37 (1975).

${ }^{10}$ S. Motojima, M. Kawaguchi, K. Nozaki, and H. Iwanaga, Appl. Phys. Lett. 56, 321 (1990).

${ }^{11}$ C. Kuzuya, Y. Hayashi, and S. Motojima, Carbon 40, 1071 (2002).

${ }^{12}$ J. Xie, P. K. Sharma, V. V. Varadan, V. K. Varadan, B. K. Pradhan, and S. Eser, Mater. Chem. Phys. 76, 217 (2002).

${ }^{13}$ Y. Qin, Z. Zhang, and Z. Cui, Carbon 41, 3072 (2003).

${ }^{14}$ D. Liu, Q. Luo, and H. Wang, Mater. Des. 30, 649 (2009).

${ }^{15} \mathrm{M}$. Weisenberger I. Martin-Gullon, J. Vera-Agullo, H. Varela-Rizo, C. Merino, R. Andrews, D. Qian, and T. Rantell, Carbon 47, 2211 (2009).

${ }^{16}$ X. Chen, S. Yang, and S. Motojima, Mater. Lett. 57, 48 (2002).

${ }^{17}$ V. V. Chesnokov, V. I. Zaikovskii, and R. A. Buyanov, J. Mol. Catal. A: Chem. 158, 267 (2000).

${ }^{18}$ M. Zhang, Y. Nakayama, and L. Pun, Jpn. J. Appl. Phys., Part 2 39, L1242 (2000).

${ }^{19}$ M. Kawaguchi, K. Nozaki, S. Motojima, and H. Iwanaga, J. Cryst. Growth 118, 309 (1992).

${ }^{20}$ W. B. Downs and R. T. K. Baker, Carbon 29, 1173 (1991).

${ }^{21}$ S. Yang, X. Chen, M. Kusunoki, K. Yamamoto, H. Iwanaga, and S. Motojima, Carbon 43, 916 (2005).

${ }^{22}$ S. Lubkin, Bull. Math. Biol. 56, 795 (1994).

${ }^{23}$ C. J. Benham, Biopolymers 22, 2477 (1983).

${ }^{24}$ N. H. Mendelson, Sci. Prog. Oxford 74, 425 (1990).

${ }^{25}$ JCPDS Card No. 23-0064.

${ }^{26}$ B. K. Gupta and O. N. Srivastava, Int. J. Hydrogen Energy 26, 857 (2001).

${ }^{27}$ Y. Yan-Hong, M. Run-Cai, B. Jin-Tao, and H. Xun, Chin. Phys. 15, 2761 (2006).

${ }^{28}$ H. B. Willard, L. L. Merritt, and J. A. Dean, Instrumental Methods of Analysis, 5th ed. (Van Nostrand, New York, 1974). 